$\begin{array}{cc}\text { ACADEMIA ROMÂNĂ } & \\ \text { Rev. Roum. Chim., } & \text { 2019, 64(6), 479-484 } \\ \text { Revue Roumaine de Chimie } & \text { http://web.icf.ro/rrch/ }\end{array}$

\title{
PREPARATION OF POLYAMIDE 6/COPPER NANOCOMPOSITES AND ITS PROPERTIES
}

\author{
Qijie XU, ${ }^{a}$ Ning ZHANG,${ }^{\mathrm{a}^{*}}$ Dandan HE, ${ }^{\mathrm{a}}$ Zhun LIU, ${ }^{\mathrm{a}} \mathrm{Wei} \mathrm{LI}^{\mathrm{a}}$ and Weiwei HE \\ ${ }^{a}$ College of Chemistry and Pharmaceutical Engineering, Huanghuai University, Zhumadian 463000, Henan, China \\ ${ }^{\mathrm{b}}$ College of advanced materials and energy, Xuchang University, Xuchang, 461000, Henan, China
}

Received July 19, 2018

Polyamide 6/copper (noted as $\mathrm{PA} / \mathrm{Cu}$ ) nanocomposites was prepared readily where $\mathrm{CuO}$ was used as the filler and adipic acid as the catalyst. $\mathrm{CuO}$ was reduced to $\mathrm{Cu}$ elemental as well as dispersed in the polymerization system of PA6 via using the reducing atmosphere of the polymerization process. As-prepared $\mathrm{PA} 6 / \mathrm{Cu}$ nanocomposites were characterized by means of differential scanning calorimetry (DSC), X-ray diffraction (XRD), ultraviolet-visible absorption spectroscopy (UV) and transmission electron microscopy (TEM). Results showed that $\mathrm{CuO}$ was reduced to $\mathrm{Cu}$ elemental, and evenly dispersed in PA6 matrix. The melting temperature of PA6 matrix material improved with increasing the content of $\mathrm{Cu}$ in comparison with pure PA6, however, the crystallization temperature tended to the low temperature. The crystal structure of $\mathrm{PA} 6 / \mathrm{Cu}$ nanocomposites tended to generate $\gamma$ crystal shape. Additionally, the size of as-fabricated nanoparticle was about $30-50 \mathrm{~nm}$, and the ultraviolet absorption of $360 \mathrm{~nm}$ represented the absorption of $\mathrm{Cu}$ nanoparticles. The mechanical properties and friction behaviors of $\mathrm{PA} 6 / \mathrm{Cu}$ nanocomposites were evaluated by means of universal tensile testing machine, impact testing machine, and friction testing machine. Results showed that as-prepared composites with $0.3 \%$ nano-Cu content possessed the best tensile strength of $27.75 \mathrm{MPa}$, as increased by $29.1 \%$ in comparison that of PA6. Furthermore, the impact toughness of PA6/Cu nanocomposites with nano-Cu content of $0.7 \%$ reached to $7.20 \mathrm{MPa}$, as increased by $170.5 \%$. Friction coefficient and grinding spot diameter presented a decreasing tendency with increasing the nano-Cu content.

\section{INTRODUCTION}

Among the exceptional property enhancements of polymer by adding fillers, such as mechanical properties, thermal stability, chemical resistance, friction properties, flame retardancy and barrier resistance, of particular interest are the improvements in tribological behavior and mechanical properties. ${ }^{1-8}$ Polymer-based composites with high strength and self-lubricity materials can be applied in seals, gears, bearings and cams that replaces the metallic materials and avoids the need for oil or grease lubrication with its attendant

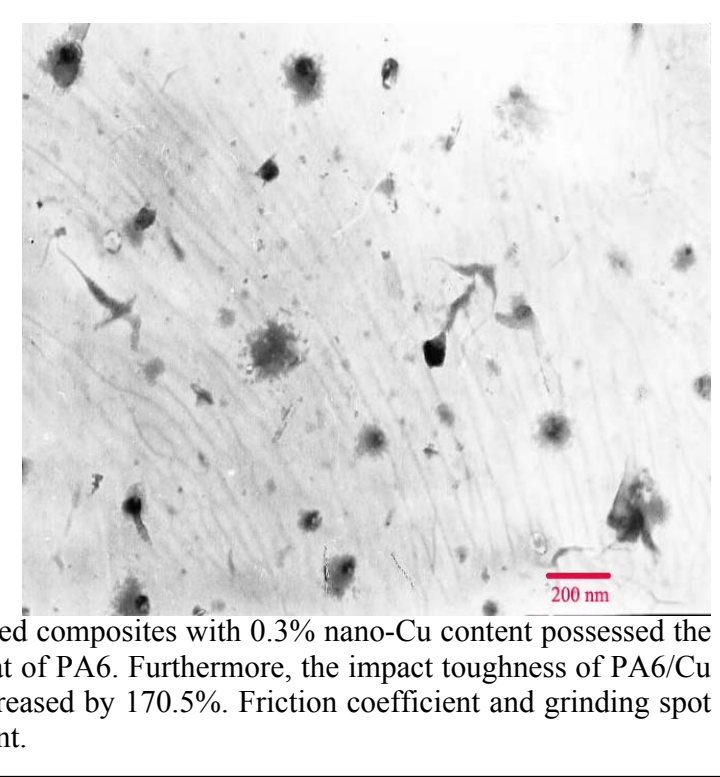

problems of sealing and possible contamination. Currently, a variety of inorganic compounds, such as $\mathrm{MoS}_{2}$, graphite, $\mathrm{CuO}, \mathrm{CuS}, \mathrm{PbO}, \mathrm{Fe}_{2} \mathrm{O}_{3}$, and metallic powders, $\mathrm{TiO}_{2}$, clay, $\mathrm{CaCO}_{3}$, nanosilica, have been used as fillers to improve the tribological behavior, mechanical property, or both. $^{2,5,9-11}$ Fibrous materials are also used for improving the properties of polymer matrix..$^{3,712}$

There is a significant challenge that is uniform dispersion of the metal fillers in polymer matrix. Particularly, there are two challenges, oxidation and aggregation of nanometal, in the preparation process of the polymer-based metal nanocomposites. A novel

\footnotetext{
*Corresponding author: qijie001@163.com; Tel./fax: +86 3962853411
} 
synthesis strategy is found that metal oxides or ions are added in polymerization system with extraneous strong reductants, therefore, polymer-based metallic nanocomposites with uniform nanometal particles are prepared. ${ }^{13,14}$ The by-product by reducing metal oxides or ions exist in the polymer matrix, moreover, may affect the properties of polymer.

Throughout the preparation process of polyamide 6 (PA6), the reaction system always remains weak reduction atmosphere due to the reduction performance of the amino groups of PA6 structure. In such the weak reducing atmosphere, some metal oxides or ions, such as $\mathrm{CuO}, \mathrm{Ag}^{+}, \mathrm{Fe}^{3+}$, and so on, may be reduced into metal elements without extraneous reductants, furtherly, which uniformly disperse in polymer matrix.

Bearing those thoughts in mind, $\mathrm{CuO}$ is chosen as a filler to fabricate $\mathrm{PA} 6 / \mathrm{Cu}$ nanocomposites, in which $\mathrm{CuO}$ will be reduced to $\mathrm{Cu}$ element via using the weak reduction atmosphere of polymerization system of PA6, and that uniformly disperse in PA6 matrix, furthermore, hoping to improve the mechanical properties and wear resistance. This paper reports the preparation as well as investigation of mechanical properties, friction and wear behavior, and thermal stability of as-prepared $\mathrm{PA} 6 / \mathrm{Cu}$ composites.

\section{EXPERIMENTAL}

\section{Materials}

The $\varepsilon$-caprolactam was obtained from BASF Co. China. Adipic acid was supplied by Paini chemical reagent factory of Zhengzhou. Formic acid was supplied by Kemel chemical reagent Co. Ltd of Tianjin. Copper oxide was obtained from Hedong district red crag reagent factory of Tianjin. Copper oxide was supplied by Nanjing Milan chemical Co., ltd. Copper sulphate $\left(\mathrm{CuSO}_{4}\right)$ was supplied by Suzhou chemical reagent factory of Tianjin.

\section{Processing}

$\mathrm{CuO}$, adipic acid, $\varepsilon$-caprolactam, and distilled water were added to a three necks flask of $250 \mathrm{~mL}$. The temperature of system was then elevated from room temperature to $210^{\circ} \mathrm{C}$ over a period of about $3 \mathrm{~h}$ with stirring. The temperature was independently elevated to $230^{\circ} \mathrm{C}$ by releasing the water vapor, and then to $260^{\circ} \mathrm{C}$ in $1.5-2 \mathrm{~h}$ gradually. Furtherly, the pressure of system was pumped to $-0.08 \mathrm{MPa}$ gradually with circulating water vacuum pump. Thereafter, the system was cooled slowly to the room temperature, and $\mathrm{PA} 6 / \mathrm{Cu}$ nanocomposites was obtained. Then the composite was injected into the model to gain dumbbell shape spline for evaluating the tensile strength of the material, "V" notched type for testing impact toughness, and block sample piece was obtained for tribology performance test.

Additionally, all samples were dried in an oven for $24 \mathrm{~h}$ at $80^{\circ} \mathrm{C}$ in order to eliminate the influence of moisture content on the properties of ceramic materials.

\section{Characterization and testing}

Differential scanning calorimetry (DSC $822^{\mathrm{e}}$, Mettler Toledo, Sweden) was used for analysis of the crystallization and melting behaviors of PA6 and PA6/Cu nanocomposites, and the samples were heated from $25^{\circ} \mathrm{C}$ to $260^{\circ} \mathrm{C}$ at the rate of $10^{\circ} \mathrm{C} / \mathrm{min}$, and then, the samples were cooled back to $25^{\circ} \mathrm{C}$. Crystal type and spherocrystal of nanocomposites of PA6 and $\mathrm{PA} / \mathrm{Cu}$ nanocomposites are investigated by X-ray diffraction (XRD), and the samples were tested in the condition of $30 \mathrm{~mA}$ in current, voltage of $40 \mathrm{kV}$, scanning speed $2 \%$ min, the scanning range is $5^{\circ}-85^{\circ}$, scan interval is $0.03^{\circ}$. Ultravioletvisible absorption spectroscopy (UV-vis) was carried out for detecting the UV-vis absorption spectra of PA6, PA6/Cu nanocomposites, in addition, the dispersion performance of nano- $\mathrm{Cu}$ in PA6 was evaluated by transmission electron microscopy (TEM). Tensile properties were measured on a WDW-10D testing machine according to GB/T 1040-1992, impact tests were measured on a ZBC-1400-2 testing machine according to GB/T 1040-1993. The friction and wear properties of PA6 and $\mathrm{PA} 6 / \mathrm{Cu}$ nanocomposites were measured by MRH-3 high speed block-on-ring test machine at the rate of $150 \mathrm{r} \cdot \mathrm{min}^{-1}$ within $1 \mathrm{~h}$. Ubbelohde viscometer was used for determining the molecular weight of PA6 and PA6/Cu nanocomposites, and the samples were dissolved in the $85 \%$ formic acid solution, with the temperature of $30^{\circ} \mathrm{C}$ water bath testing its viscosity, using extrapolation method to find out the corresponding samples corresponding characteristic viscosity, and then molecular weight were calculated. Computation formula is as follows:

$$
[\eta]=K \overline{M \eta}^{a}
$$

Among them, $K$ is equal to $2.26 \times 10^{-3}$, and $\alpha$ is equal to 0.82 according to macromolecular chemistry experiment. ${ }^{15}$

\section{RESULTS AND DISCUSSION}

\section{The characterization of PA6 and PA6/Cu nanocomposites}

Usually, the crystallization and melting performance of PA6 and $\mathrm{PA} 6 / \mathrm{Cu}$ nanocomposites are evaluated by using DSC. Figure 1 showed that the cooling temperature $\left(T_{c}\right)$ and the melting temperature $\left(T_{m}\right)$ of PA6 and PA6/Cu nanocomposites. As could be seen in Figure 1A, $T_{c}$ of nanocomposites tended to gradually decrease with increasing the nano- $\mathrm{Cu}$ content, and $\chi_{c}$ also appear a reducing trend. However, melting curves, as seen in Figure 2B, presented a molten twin peaks that may contain $\alpha$ crystal and $\gamma$ crystal, moreover, $T_{m}$ and the shape of the molten peak of PA6 was the same as those of PA6/Cu nanocomposites, which showed that the addition of nano-copper has little influence on the crystalline form of PA6.

Other DSC parameters, including melting enthalpy $\left(\Delta H_{m}\right)$, cooling enthalpy $\left(\Delta H_{c}\right)$, crystallinity $\left(\chi_{c}\right)$, and the peak width at half height $\left(\Delta T_{c l / 2}\right)$, were showed in Table 1 . It was seen that $\Delta H_{m}$ presented a rising tendency with increasing the nano-Cu content, and $\Delta H_{c}$ showed a reducing trend that corresponded 
to the trend of $\chi_{c}$ of PA6 and PA6/Cu nanocomposites.

The possible reasons were as follow: (1) the interactions of nano-copper and PA6 limited the movement of the molecular chains of PA6, moreover, hindered the crystallization process of PA6 matrix, which leads to a decreasing trend of the crystallinity and half peak width, (2) the heterogeneous nucleation of nano-copper improved the crystallization rate of
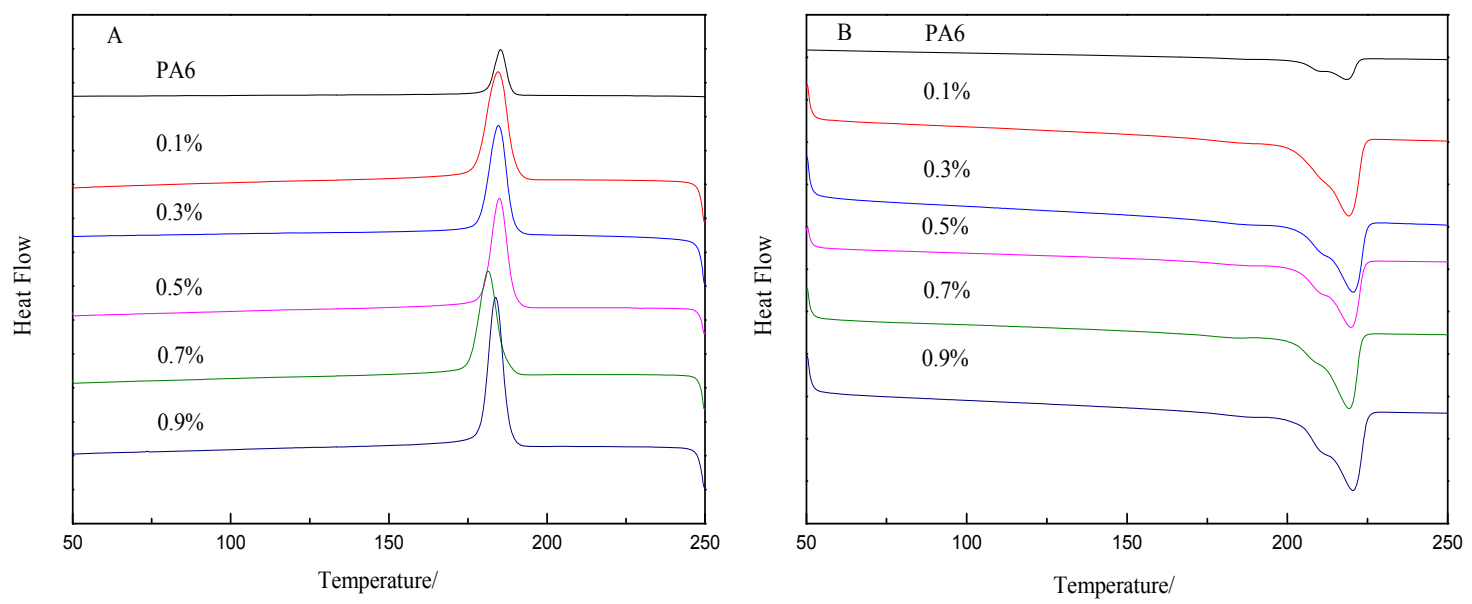

Fig. 1 - Cooling curves (A) and melting curves (B) of PA6 and PA6/Cu nanocomposites.

Table 1

The DSC parameters of PA6 and PA6/Cu nanocomposites

\begin{tabular}{lllllll}
\hline Samples & $\begin{array}{l}\boldsymbol{T}_{\boldsymbol{m}} \\
\left({ }^{\mathbf{0}} \mathbf{C}\right)\end{array}$ & $\begin{array}{l}\boldsymbol{T}_{\boldsymbol{c}} \\
\left({ }^{\mathbf{0}} \mathbf{C}\right)\end{array}$ & $\begin{array}{l}\Delta \boldsymbol{H}_{\boldsymbol{m}} \\
(\mathbf{J} / \mathbf{g})\end{array}$ & $\begin{array}{l}\Delta \boldsymbol{H}_{\boldsymbol{c}} \\
(\mathbf{J} / \mathbf{g})\end{array}$ & $\begin{array}{l}\chi_{c} \\
(\mathbf{\%})\end{array}$ & $\begin{array}{l}\Delta \boldsymbol{T}_{\boldsymbol{c l} / \mathbf{2}} \\
(\mathbf{m i n})\end{array}$ \\
\hline PA6 & 219.52 & 185.38 & 54.02 & 60.68 & 26.38 & 0.39 \\
PA6/Cu-0.1g & 218.78 & 185.00 & 65.92 & 58.06 & 25.24 & 0.36 \\
PA6/Cu-0.3g & 220.08 & 185.17 & 68.60 & 64.00 & 27.83 & 0.31 \\
PA6/Cu-0.5g & 219.55 & 185.46 & 69.10 & 57.69 & 25.08 & 0.28 \\
PA6/Cu-0.7g & 218.98 & 181.79 & 66.99 & 55.98 & 24.34 & 0.32 \\
PA6/Cu-0.9g & 220.10 & 184.47 & 74.81 & 56.02 & 24.36 & 0.26 \\
\hline
\end{tabular}

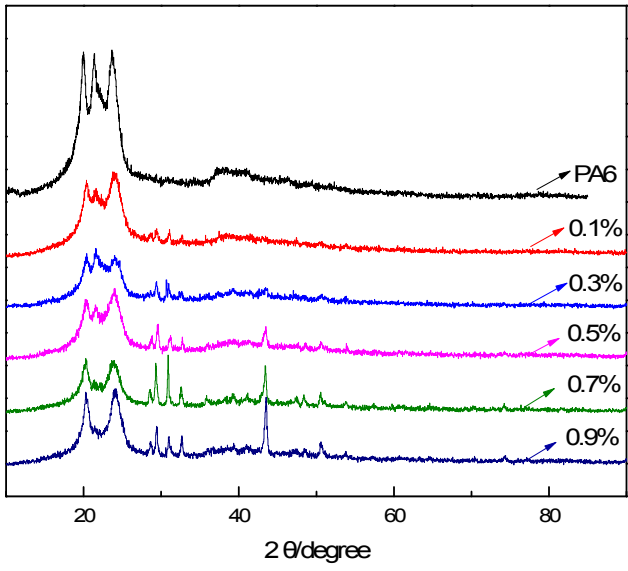

Fig. 2 - XRD of PA6 and PA6/Cu nanocomposites.
PA6, (3) the bigger size of the crystals in the substrate material led to the greater the degree of crystallization, the more perfect of crystalline, and the higher the melting temperature and melting enthalpy. Therefore, limiting the movement of the molecular chains of PA6 and the strong heterogeneous nucleation ability of nano- $\mathrm{Cu}$ were the main factors influencing the properties of $\mathrm{PA} 6 / \mathrm{Cu}$ nanocomposites. $^{13-15}$ 
Figure 2 showed the XRD patterns of PA6 and $\mathrm{PA} 6 / \mathrm{Cu}$ nanocomposites. As could be seen that PA6 possessed $\alpha$ crystal corresponding to $19.9^{\circ}$ and $23.8^{\circ}$ and $\gamma$ crystal of $21.38^{\circ}$. However, with increasing content of nano- $\mathrm{Cu}, \gamma$ crystal, as imperfect crystal, tended to a decreasing tendency, even $\gamma$ crystal in $\mathrm{PA} 6 / \mathrm{Cu}$ nanocomposite disappeared while content of nano-copper reached to $0.9 \%$. The possible reason was heterogeneous nucleation of nano-copper that could improve the crystal rate, thus led to convert $\gamma$ crystal to $\alpha$ crystal. ${ }^{12-16}$

Additionally, the absorption peaks of $43.3^{\circ}$, $50.1^{\circ}, 74.1^{\circ}$, and $89.9^{\circ}$ were good agreement with reflections of $\mathrm{Cu}$ elemental, ${ }^{12}$ which indicated $\mathrm{Cu}$ nanoparticles was formed from $\mathrm{CuO}$ in the process of PA6 polymerization by in situ method, moreover, it was not oxidized in the high temperature reaction process. There was because that the amino group of PA6 structure showed weak reducibility that keep the reducing atmosphere in PA6 polymerization process system. Furthermore, $\mathrm{Cu}$ nanoparticles was coated with PA6 molecular chain that formed a layer of protective film to prevent the influence of air and moisture. Thus, PA6/Cu nanocomposite could keep its original state and properties in nature environment for a long time ${ }^{[16]}$.

Figure 3 showed the TEM image of $\mathrm{PA} 6 / \mathrm{Cu}$ nanocomposites with nano-Cu content of $0.5 \%$ that possessed perfect mechanical properties. As could be seen that the size of nano-Cu was about $30-50 \mathrm{~nm}$, and coated by PA6 matrix, moreover, uniformly dispersed in PA6 matrix. Thus, forming a layer of protective film around nano- $\mathrm{Cu}$ was helpful to prevent aggregation, oxidation of nano- $\mathrm{Cu}$.

\section{The mechanical properties of PA6 and $\mathrm{PA6} / \mathrm{Cu}$ nanocomposite}

Figure 4 showed the effect of nano-Cu content on tensile strength and impact toughness of PA6. It was seen that tensile strength of PA6 with nano- $\mathrm{Cu}$ content of $0.3 \%$ possessed the maximum value that reached $27.75 \mathrm{MPa}$, improved $29.1 \%$ as comparing with that of PA6. For impact toughness of PA6, the maximum value was $7.20 \mathrm{MPa}$ while nano-Cu content was $0.7 \%$, as improving $170.5 \%$. Both tensile strength and impact toughness of PA6/Cu nanocomposites had a similar trend that increased with increasing nano- $\mathrm{Cu}$ content and then started to decrease. The main reason was concluded that nano- $\mathrm{Cu}$ uniformly dispersed in PA6 matrix while the nano-Cu content was lower than $0.3 \%$. However, the viscosity of the system increased with continuing to increase the nano- $\mathrm{Cu}$ content, which hindered the movement of PA6 molecular chains and then led to reducing the molecular weight of $\mathrm{PA}^{17}{ }^{17}$ Additionally, the aggregation of nano-Cu may occur with increasing nano- $\mathrm{Cu}$ content that easily formed stress concentration point and then reduced the mechanical properties of $\mathrm{PA} 6^{[18]}$.

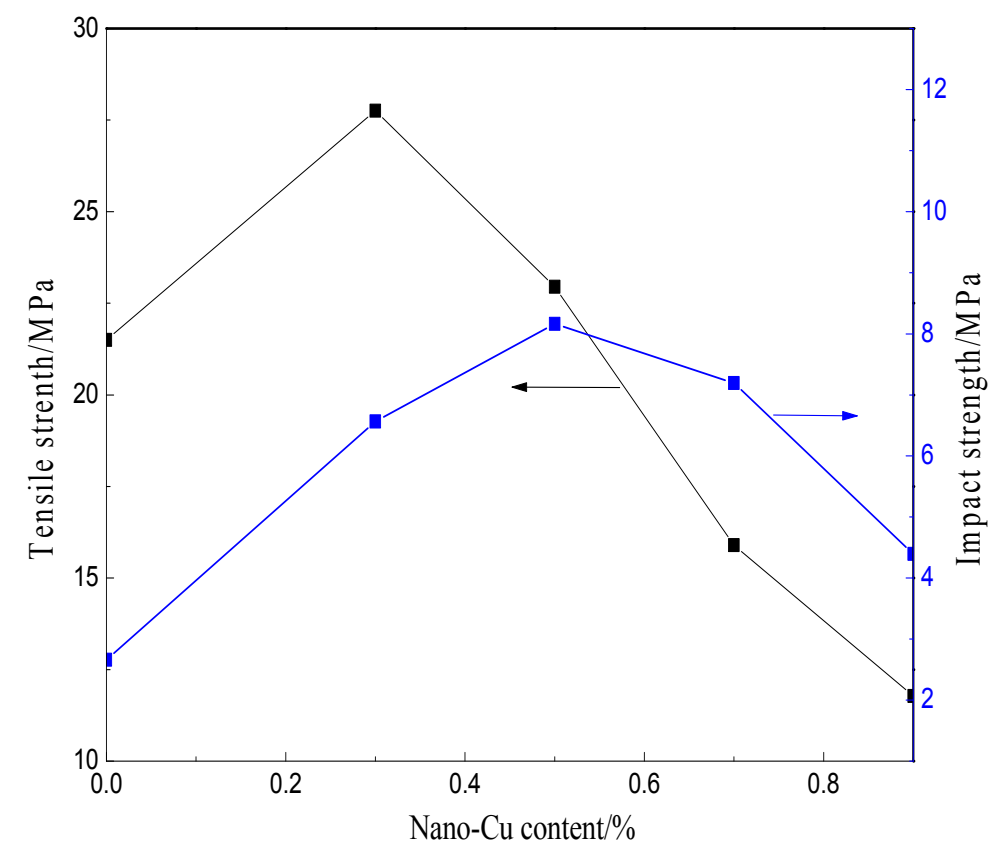

Fig. 4 - The tensile strength and impact strength of PA6 and PA6/Cu nanocomposites. 

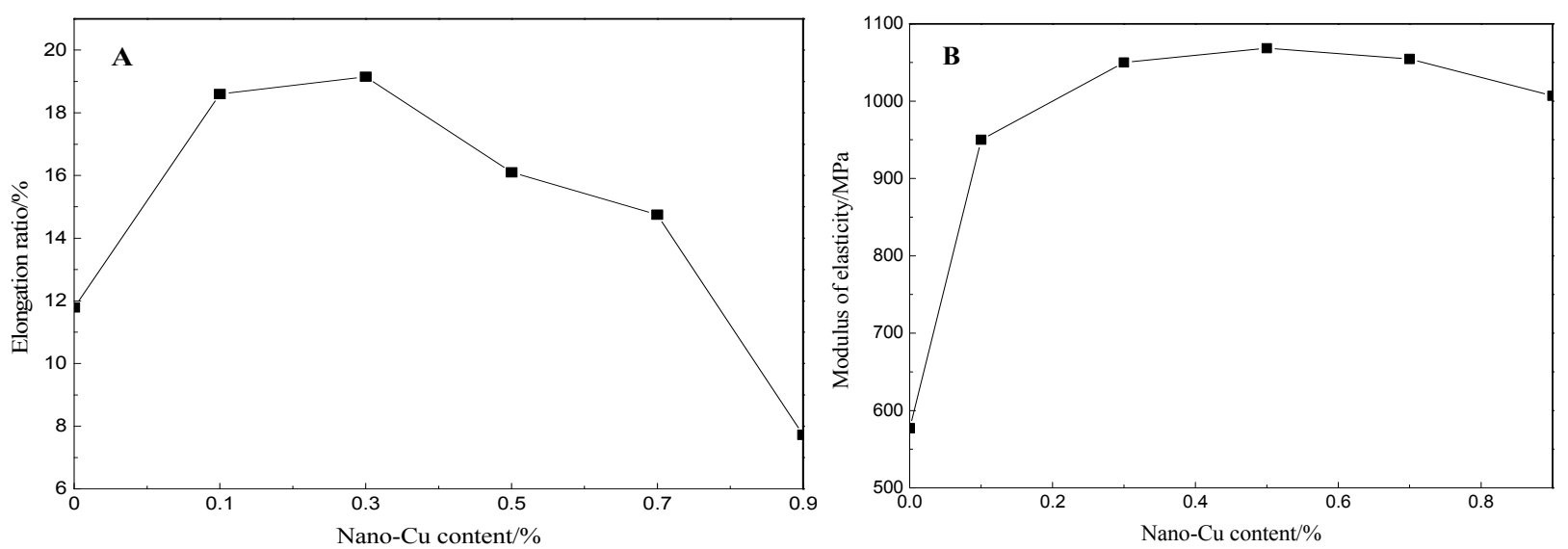

Fig. 5 - The elongation ratio (A) and elasticity modulus (B) of PA6 and PA6/Cu nanocomposite.

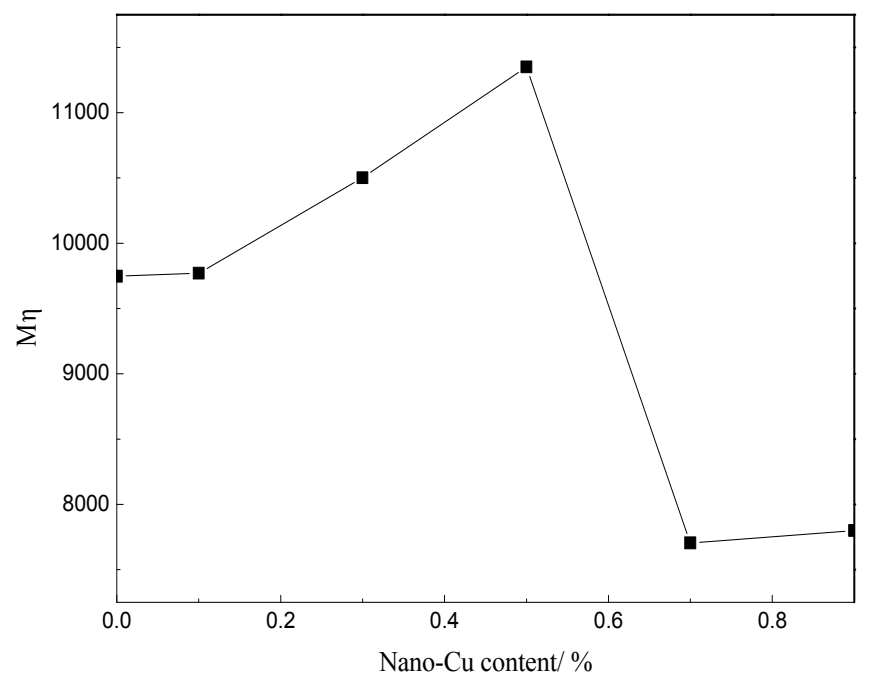

Fig. 6 - Viscosity average molecular weight of PA6 and PA6/Cu nanocomposites.
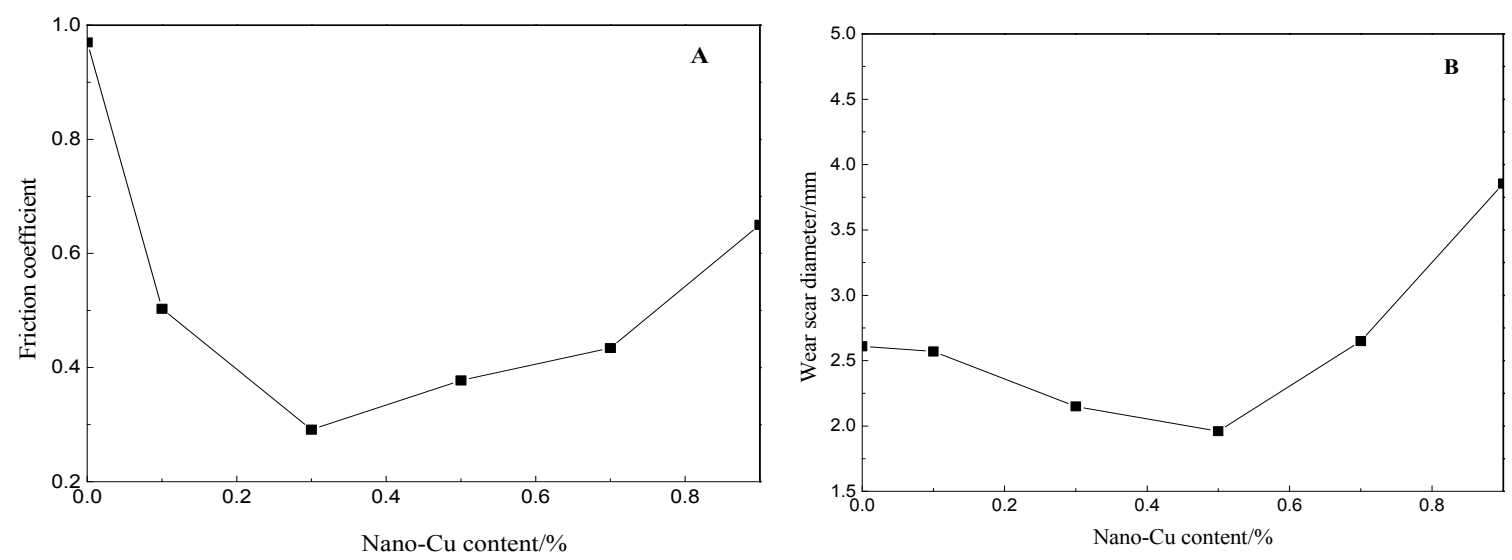

Fig. 7 - Friction coefficient (A) and wear scar diameter (B) of PA6 and PA6/Cu nanocomposites.

Figure 5 showed the effect of nano-Cu on the elongation at break (Figure $5 \mathrm{~A}$ ) and the elastic modulus (Figure 5B) of PA6. It could be seen that the elongation at break of $\mathrm{PA} 6 / \mathrm{Cu}$ nanocomposites with nano- $\mathrm{Cu}$ content of $0.3 \%$ had the maximum value of $19.15 \mathrm{~mm}$, as improving $62.4 \%$ with comparing that of PA6, and then presented a gradually decreasing trend. Additionally, the elastic modulus indicated a rising trend with increasing nano- $\mathrm{Cu}$ content. The maximum elastic modulus reached to $1068 \mathrm{MPa}$ while the nano- $\mathrm{Cu}$ content was $0.7 \%$ and improved $85.4 \%$. 
The reasons may conclude as follow: (1) the external strain between nano- $\mathrm{Cu}$ and PA6 made the molecular chains move in the form of a scratch, in addition, nano- $\mathrm{Cu}$ was regarded as spheroidal structure that is benificial for reducing the interaction between PA6 molecular chains. Thus, nano- $\mathrm{Cu}$ could improve the elongation at break of PA6. (2) Due to Nano-copper had perfect elastic modulus, $\mathrm{PA} 6 / \mathrm{Cu}$ nanocomposite possessed greater elastic modulus, moreover, the strong interaction between nano- $\mathrm{Cu}$ and PA6 and heterogeneous nucleation of nano-Cu enabled PA6 to form perfect crystals, thereby increasing the elastic modulus. ${ }^{19,20}$

Viscosity average molecular weight of PA6 and PA6/Cu nanocomposites also confirmed the effect of nano- $\mathrm{Cu}$ on mechanical properties of PA6, as shown in Figure 6. With increasing nano-Cu content, the molecular weight of PA6 firstly showed the increasing trend and then decreased, moreover, $\mathrm{PA} 6 / \mathrm{Cu}$ nanocomposites with nano-Cu content of $0.5 \%$ had the largest molecular weight, and Viscosity average molecular weight started to decrease while the content exceeded $0.5 \%$. This was mainly because that the lower content nano-Cu was banificial to form heterogeneous nucleation and improved crystallinity of PA6, or the higher content nano-Cu could aggregation and hindered the movement of PA6 molecular chains and reduced the contact probability between the active groups concluded end amino and carboxyl groups. ${ }^{17,18}$

\section{Tribological properties of PA6 and PA6/Cu nanocomposite}

Figure 7 presented the friction coefficient (Figure $7 \mathrm{~A}$ ) and wear scar diameter (Figure 7B) of PA6 and $\mathrm{PA} 6 / \mathrm{Cu}$ nanocomposites under the experimental force of $100 \mathrm{~N}$, those tend to gradually decrease with low nano- $\mathrm{Cu}$ content. Because of perfect dispersion of nano- $\mathrm{Cu}$ in PA6 matrix with low nano- $\mathrm{Cu}$ content, nano- $\mathrm{Cu}$ was benificial to reduce the adhesion strength between polymer and grinding contact area, thereby reducing the friction coefficient and wear scar area. ${ }^{12}$

\section{CONCLUSION}

PA6/Cu nanocomposites with perfect mechanical properties and tribological properties were prepared readily with in situ polymerization method, in which $\mathrm{CuO}$ was used as filler and reduced into nano- $\mathrm{Cu}$ with uniform dispersion in PA6 matrix by using weak reducing atmosphere of the end amino groups of PA6. Nano-Cu was benificial to form $\alpha$ crystal structure that could improve the mechanical properties of PA6. As compared with PA6, tensile strength of $\mathrm{PA} 6 / \mathrm{Cu}$ with nano-Cu of $0.3 \%$ improved $29.1 \%$; the impact toughness raised $170 \%$ while the amount of nano- $\mathrm{Cu}$ was $0.7 \%$. Additionally, $\mathrm{PA} 6 / \mathrm{Cu}$ nanocomposites also displayed good antiwear properties and antifriction performance.

Acknowledgements. This research is financially supported by the project of youth backbone teachers of Henan province (grant No. 2017GGJS172), Henan Provincial Natural Science Foundation of China (grant No.182102311053), and 2018's Open topic on key laboratory of nanoscale energy storage and conversion materials in Henan province (2018ISMNM01).

\section{REFERENCES}

1. W. Liu, S. W. Lee, D. Lin, et al. Nature Energy, 2017, $2,1$.

2. Y. J. Wan, W. H. Yang, S. H. Yu, et al. Composit. Sci. Techn., 2016, 122, 27.

3. V. Dhand, G. Mittal, K. Y. Rhee, et al. Composit. Part B: Eng., 2015, 73, 166.

4. S. Qiu, Y. Hou, W. Xing, et al. Chem. Eng. J., 2018, 349, 223.

5. G. Si, D. Li, Y. You Y, et al. Polym. Composites, 2017, 38, 2090.

6. H. Hirani, "Fundamentals of Engineering Tribology with applications", Cambridge University Press, 2016.

7. D. K. Jesthi, P. Mandal, A. K. Rout, et al. Procedia Manufacturing, 2018, 20, 536.

8. E. M. Afifi, A. B. Elshalakny, T. A. Osman, et al. Fullerenes, Nanotubes and Carbon Nanostructures, 2018, 26, 351.

9. K. Zhou, J. Liu, W. Zeng, et al. Composit. Sci. Techn., 2015, 107,120 .

10. T. R. Kuech, R. J. Hamers and J. A. Pedersen, "Chemical transformation of metal, metal oxide, and metal chalcogenide nanoparticles in the environment", Wiley, 2016.

11. M. A. Aldoasri, S. Darwish, M. Adam, et al. Mater. Sci., 2018, in press

12. Q. Xu Q, Li X, S. Zhang, et al. J. Polym. Research, 2013, $20,257$.

13. Z. Liu, Q. Rong, Z. Ma, et al. Biosensors and Bioelectronics, 2015, 65, 307.

14. Q. Xu, X. Feng, J. Liu, et al. Polish J. Chem. Techno., 2017, 19,36 .

15. H. Liao and J. Lu, "Macromolecular chemistry experiment", Chemical Industry Press, 2014.

16. X. Liu and Q. Wu, Eur. Polym. J., 2002, 38, 138.

17. B. Schartel, P. Pötschke, U. Knoll et al. Eur. Polym. J., 2005, $41,1061$.

18. R. S. Andre, A. Pavinatto, L. A. Mercante, et al. RSC Advances, 2015, 5, 73875 .

19. A. M. Peres, R. R. Pires and R. L. Oréfice, Carbohydrate Polym., 2016, 136, 210.

20. N. Saba, M. T. Paridah and M. Jawaid, Constr. Building Mat, 2015, 76, 87. 
